\title{
APORTE AL CONOCIMIENTO DE MACROCYSTIS PYRIFERA: REVISIÓN BIBLIOGRÁFICA SOBRE LOS "HUIRALES" DISTRIBUIDOS EN LA REGIÓN DE MAGALLANES.
}

\author{
CONTRIBUTION TO THE KNOWLEDGE OF MACROCYSTIS PYRIFERA: BIBLIOGRAPHIC \\ REVIEW OF THE KELP FORESTS DISTRIBUTED IN THE MAGELLAN REGION.
}

Carlos Ríos ${ }^{1} \&$ Erika Mutschke ${ }^{2}$

Macrocystis pyrifera (L.) C. Agardh, es una macroalga de nombre común "huiro", con una distribución geográfica de tipo bipolar y antitropical y, en la región de Magallanes, es un elemento característico en prácticamente toda la extensión de fiordos y canales tanto fueguinos como patagónicos, específicamente en sectores relativamente someros (Dayton 1974, 1985, Searles et al. 1974). Por el lado Atlántico, la especie se distribuye entre península Valdés hasta Tierra del Fuego (Kühnemann 1970) mientras que, por el lado Pacífico de la costa de Chile, alcanza hasta Valparaíso aunque también ha sido reportado en la costa del Perú (Hoffmann \& Santelices 1997).

Tempranamente, Darwin (1842 en Arnaud 1974) llamó la atención acerca de las particularidades ecológicas de esta especie en la región magallánica, señalando: "The number of living creatures of all orders whose existente intimately depends on the kelp is wonderful. A great volume might be written describing the inhabitants of one of these beds of sea-weed. Almost all the leaves, excepting those that float on the surface, are so thickly incrusted with corallines as to be of white colour. We find exquisitely delicate structure, some inhabited by simple hydra-like polypi, others by more organized kinds, and beautiful compound Ascidiae. On the leaves, also, various patelliform shells, Trochi, uncovered Molluscs, and some bivalves, are attached. Innumerable crustacea frequent every part of the plant. On shaking the great entangled roots, a pile of small fish, shells, cuttle-fish, crabs of all orders, sea-eggs, star-fish, beautiful Holothuriae, Planariae, and crawling nereidous animals of a multitude of forms, all fall out together. Often as I recurred to a branch of the kelp, I never failed to discover animals of new and curious structures. (...) I can only compare these great aquatic forests of the southern hemisphere with the terrestrial ones in the intertropical regions. Yet if in any country a forest was destroyed, I do not believe nearly so many species of animals would perish as would here from the destruction of the kelp". Pese a estas notables particularidades, a las cuales se suma la potencial importancia comercial que tiene la especie (e.g. Mansilla 2006), los estudios tanto

1 Dirección de Programas Antárticos, Universidad de Magallanes, Punta Arenas, Chile. carlos.rios@umag.cl

2 Laboratorio de Ecología y Ciencias Ambientales, Instituto de la Patagonia, Universidad de Magallanes, Punta Arenas, Chile. 
relacionados con la macroalga per se como con los ensambles de organismos asociados a sus diferentes estructuras son escasos (Véase Santelices 1992, Ríos et al. 2007), característica que es recurrente cuando se analiza el estado del conocimiento sobre los ecosistemas marinos de la región de Magallanes (Véase Arntz 1999).

En esta nota se entrega una revisión de los principales aportes al conocimiento de Macrocystis pyrifera distribuida en la región magallánica, sobre un total de 21 publicaciones directamente relacionadas con la especie y que, básicamente, tienen su punto de partida con el estudio de Santelices, entre otros autores, realizado en los "huirales" de M. pyrifera localizadas en Puerto Toro, isla Navarino, canal Beagle (Santelices $1981^{1}$. Al respecto véase revisión de Santelices 1992). El bajo número de estudios realizados hasta la fecha justifica una revisión general y no separada en niveles de análisis, aunque básicamente se pueden distinguir estudios de distribución geográfica y otros con una mayor orientación ecológica.

\section{Información disponible}

A partir de estudios de campo realizados en 1965, Alveal et al. (1973) señalan la presencia característica de Macrocystis pyrifera en aguas del estrecho de Magallanes, particularmente en lugares protegidos, formando densos "mantos" que favorecen el desarrollo de subasociaciones especiales integradas por algas de pequeña talla y por una comunidad de moluscos y crustáceos. Estos huirales aparecen preferentemente entre los 4 - 15 m de profundidad. Esta característica ha sido confirmada por Santelices (1989) quien señala que la distribución de la especie parece estar restringida a ambientes protegidos, con presencia de un sustrato conformado ya sea por roca madre o por los típicos bloques y cantos de origen glaciar. Su distribución batimétrica estaría limitada a los 8-10 m, produciendo huirales de aproximadamente $30-40 \mathrm{~m}$ de ancho. Este rango de distribución batimétrica también ha sido encontrado para poblaciones presentes en la zona argentina de isla Tierra del Fuego, canal Beagle, por

Santelices, B. 1981. Biología y factibilidad de utilización de praderas de Macrocystis pyrifera en Puerto Toro, isla Navarino, Chile. Informe Final de circulación limitada. Lab. de Zoología, Depto. de Biología Ambiental y de Poblaciones, P. Univ. Católica de Chile. 686 pp.
Adami \& Gordillo (1999) y en sectores del estrecho de Magallanes por Ríos et al. (2007).

Dentro de su rango de distribución batimétrica y particularmente para las poblaciones localizadas al sur-este del canal Beagle, Santelices \& Ojeda (1984) estudiaron la dinámica poblacional de los huirales en Puerto Toro, isla Navarino. En este sector, las densidades de ejemplares considerados como juveniles (ejemplares con menos de $1 \mathrm{~m}$ de largo) están inversamente correlacionadas con las que presentan las plantas adultas. La parte somera de su distribución coincide con un cinturón de la macroalga Lessonia vadosa, lo cual sugiere que su límite superior estaría determinado por competencia intraespecífica con esta especie, mientras que el límite de distribución inferior estaría determinado por la disponibilidad de un sustrato apropiado para el asentamiento y crecimiento de los individuos (roca madre y/o bloques y cantos). La distancia entre individuos tuvo una directa correlación con el tamaño del grampón y con el largo total de los individuos de $M$. pyrifera. Es interesante notar que estos autores demostraron un efecto significativo de la remoción del dosel flotante sobre la densidad de los juveniles de $M$. pyrifera. En áreas sin remoción del dosel la densidad varió entre $2-5$ ind $\mathrm{m}^{-2}$, con una distancia promedio entre individuos de $0,9 \mathrm{~m}$, mientras que, en áreas con remoción del dosel, la densidad de juveniles varió entre $5-15$ ind $\mathrm{m}^{-2}$, con una distancia promedio entre ellos de 0,4 m. Dayton (1985) indica densidades medias que varían entre $0,02-0,54$ ind juveniles $\mathrm{m}^{-2} \mathrm{y}$ entre $0,02-0,94$ ejemplares adultos $\mathrm{m}^{-2}$ para sectores cercanos al estrecho de Magallanes, específicamente en áreas relativamente protegidas al oleaje oceánico, pero estas densidades aumentan tanto hacia el sur como hacia el norte del Estrecho. La diferenciación en relación a las densidades a lo largo de un gran gradiente entre la isla de los Estados por el Atlántico hasta el golfo de Corcovado en Chiloé, estaría influenciada por el grado de exposición al oleaje, el pastoreo por el erizo Loxechinus albus e, indirectamente, por efecto de la pesquería sobre L. albus (Dayton 1985).

Santelices \& Ojeda (1984) estudiaron la estratificación de la vegetación relacionada con $M$. pyrifera en sectores al sur del canal Beagle, Chile. El primer estrato vegetacional está representado por el dosel flotante de M. pyrifera, mientras que un segundo estrato aparece dominado por ejemplares 
de Lessonia flavicans, con la cual comparte la mayor parte del huiral. Existe además un tercer estrato conformado por algas frondosas siendo las más frecuentes y las más relevantes según su biomasa, Gigartina skottsbergii y Epymenia falklandica.

En los estudios realizados para caracterizar la fauna asociada a los huirales de $M$. pyrifera en la región de Magallanes, los grampones o discos de fijación al sustrato de la macroalga aparecen como los microhábitats más importantes para una amplia variedad de especies de vertebrados e invertebrados (e.g. Santelices \& Ojeda 1984, Adami \& Gordillo 1999, Cariceo et al. 2003, Ríos et al. 2007). En las frondas, Adami \& Gordillo (1999) encontraron una dominancia de moluscos, particularmente del bivalvo incubador Gaimardia trapesina y de ramoneadores como el anfípodo Paramphitoe femorata. Ojeda \& Santelices (1984) reportaron la presencia de 42 taxa de invertebrados y una especie de pez asociados a los grampones de las macroalgas presentes en Puerto Toro, isla Navarino. Los valores máximos de densidad y de biomasa se encontraron en invierno tardío y primavera, con valores máximos de riqueza de especies y diversidad ( $\left.\mathrm{H}^{\prime}\right)$ en invierno y mínimos en verano. Las especies más importantes en esta zona, por su efecto en los cambios en densidad y en biomasa, fueron Pseudechinus magellanicus, Anasterias antarctica (equinodermos), Pagurus forceps y Halicarcinus planatus (crustáceos decápodos). Estos autores sugieren que el grampón no jugaría un rol importante como espacio para el reclutamiento de especies y que las diferencias significativas en riqueza de especies y en diversidad en comparación con los valores determinados para bosques de $M$. pyrifera del hemisferio norte (e.g. en California en donde se han encontrado sobre 100 taxa de invertebrados [Ojeda \& Santelices 1984]), se deberían a causas históricas y biogeográficas.

En la zona argentina del canal Beagle (Ushuaia), Adami \& Gordillo (1999) indican la presencia de 68 taxa presentes en el sistema formado por $M$. pyrifera, incluyendo algas (5), poríferos (indeterminados), briozoos (7), nemertinos (32), poliquetos (10), moluscos (22), crustáceos (15) y equinodermos (7). Los grampones de $M$. pyrifera muestran una mayor diversidad de taxa en comparación con las frondas, con una dominancia de poliquetos, seguido por moluscos. Según el valor de H', en este sector la mayor diversidad se observó en el período de otoño-invierno, con un predominio de especímenes juveniles en primavera-verano. Adami \& Gordillo (1999) sugieren que, en este caso, los grampones pueden constituir áreas específicas para el reclutamiento de juveniles, especialmente para moluscos. Un aspecto similar fue sugerido para el echinoideo Pseudechinus magellanicus, especie común en los grampones del estrecho de Magallanes (Ríos et al. 2003).

Estudios realizados en el estrecho de Magallanes por Cariceo et al. (2003) y Ríos et al. (2007) evidenciaron una mayor riqueza de especies de invertebrados y vertebrados asociados a los grampones de M. pyrifera. En el estudio realizado en dos sectores localizados en los extremos del Paso Ancho del Estrecho (fuerte Bulnes y bahía Laredo), se registraron 114 especies faunísticas pertenecientes a 10 taxa. El grupo dominante fue poliquetos con 43 especies/géneros (especies dominantes Platynereis australis, Hermadion magalhaensis, Policyrrus sp.), seguido por artrópodos con 21 especies/géneros de crustáceos (dominantes Dynamenella eatoni, Exosphaeroma lanceolada, Halicarcinus planatus) y 1 de pantópoda (Picnogonido Indet), moluscos con 19 especies/géneros (dominantes Mytilus chilensis, Hiatella solida, Aulacomya ater), equinodermos con 12 especies/géneros (dominantes Ophiactis asperula, Pseudechinus magellanicus, Anasterias antarctica), 10 especies de peces (dominantes $P_{O}$ gonolychus marinae, Phucocoetes latitans, Maynea microphtalmus, Patagonotothen cornucola), 4 especies/géneros de cnidarios (dominante Anthothoe cf. chilensis) y, finalmente, nemertinos, sipuncúlidos, braquiópodos y ascidias, todos registrados con una especie. Una alta dominancia de especies de poliquetos se observó en ambas áreas de estudio, seguidos por crustáceos, moluscos y equinodermos. La riqueza de especies encontrada en este sector de la región de Magallanes podría ser mayor aún si se considera que varios taxa no pudieron ser identificados a nivel de especie (e.g. anfípodos, ascideas) $y$, otros, no fueron considerados en los análisis del estudio por corresponder a especies coloniales (e.g. briozoos y poríferos). Los valores máximos de riqueza de especies y abundancias fueron encontrados en los períodos de otoño-invierno, aspecto que fue consistente en ambas áreas estudiadas. La variación estacional en las abundancias y en número de especies fue relacionada con los disturbios que 
pueden producir en los ejemplares de $M$. pyrifera los fuertes vientos (y parámetros asociados como oleajes y mareas), los cuales son predominantes en las estaciones de primavera y verano austral. Las diferencias observadas en la estructura comunitaria también podrían ser explicadas en función de la heterogeneidad en las condiciones ambientales de la región de Magallanes, lo cual sugiere una importante heterogeneidad en la estructura de las comunidades y en las dinámicas poblacionales de los organismos asociados con $M$. pyrifera.

Un grupo específico asociado a los huirales en el sur del canal Beagle es el de los equinodermos, específicamente erizos, estudiados por Vásquez et al. (1984). En total se registraron cuatro especies de erizos (Loxechinus albus, Pseudechinus magellanicus, Arbacia dufresney, Austrocydarus canaliculata) asociados a cuatro tipos de microhábitats definidos en los huirales estudiados. Las mayores abundancias de estas especies fueron encontradas sobre y debajo de bloques presentes en el huiral, en donde además fueron más frecuentes. Las especies más abundantes fueron Pseudechinus magellanicus y Loxechinus albus. Dos especies fueron encontradas en los cuatro microhábitats (Pseudechinus magellanicus y Austrocidarus canaliculata) pero con diferentes probabilidades. La primera de ellas ocurre más frecuentemente dentro de los grampones de $M$. pyrifera mientras que la segunda es más frecuente bajo los bloques. L. albus fue encontrada principalmente sobre bloques y con menor frecuencia en grietas dentro del huiral, aunque nunca dentro de los grampones. A partir del análisis del contenido estomacal de estas especies, se concluyó que ellas están depredando sobre cuatro grupos principales de alimentos (frondas de $M$. pyrifera, otras algas frondosas, algas calcáreas e invertebrados). Las cuatro especies se alimentan de $M$. pyrifera pero en diferentes porcentajes aunque el recurso alimentario parece no ser limitante para ellas. Los patrones de distribución en los microhábitat y las dietas de los erizos sugieren que no existe una sobreposición en la utilización del espacio por parte de las cuatro especies, excepto para el par $P$. magellanicus- $A$. canaliculata.

Otro grupo importante asociado a los huirales de $M$. pyrifera son los peces, predominantemente Notothenidae, los cuales también presentan diferencias importantes en términos de su abundancia y diversidad según sean las áreas que se analicen. Vanella et al. (2007) señalan que las diferencias en diversidad respecto de los resultados obtenidos por Moreno \& Jara (1984) podrían estar asociadas con la distancia a la costa de los correspondientes huirales. En huirales relativamente más alejados de la costa, Vanella et al. (2007) encontraron 11 especies, mientras que Moreno \& Jara (1984) reportaron 18 especies en huirales más cercanos, con una mayor representación de especies que también se distribuyen hacia la zona intermareal (e.g. Harpagifer bispinis). En el estudio de Moreno \& Jara (1984) sólo una especie (Patagonotothen sima) fue asociada al grampón de M. pyrifera, producto de una migración de tipo reproductiva durante el período invernal. Las restantes especies ocupan diferentes niveles de la columna de agua dentro del huiral. Vanella et al. (2007) encontraron 6 especies dentro de los grampones, siendo el nototénido Patagonotothen cornucola la especie dominante en este microhábitat. En el estudio de Adami \& Gordillo (1999) no fueron recolectados peces durante el trabajo de un año realizado en el canal Beagle, Tierra del Fuego, mientras que Ríos et al. (2007) encontraron 10 especies asociadas a los grampones de $M$. pyrifera, incluyendo un primer registro del zoarcido Crossostomus sobrali para el área del estrecho de Magallanes (Morrison \& Pequeño 2003). Moreno \& Jara (1984) determinaron que el conjunto de peces asociados a $M$. pyrifera en el sur del Beagle no consumen directamente a la macroalga ni a erizos.

Dentro de los depredadores más efectivos en los huirales, Vásquez \& Castilla (1984) mencionan al asteroideo Cosmasterias lurida, que aparece asociada a los sustratos duros al interior del huiral o fuera de éste. La dieta de C. lurida incluye 25 especies de moluscos, crustáceos, ascidias, peces y braquiópodos, y no se encontraron evidencias de selectividad por ninguna de las especies lo cual sugiere una condición de generalista para $C$. lurida que consume de acuerdo a la abundancia local de las presas. Desde un punto de vista trófico, Castilla (1985) sugiere que ninguna de las especies de depredadores incluidas dentro de la fauna asociada a $M$. pyrifera (e.g. el gastrópodo Fusitriton magellanicus, el anatidae Tachyeres pteneres) podría ser considerada una especie clave dentro del sistema. A partir de estudios realizados en un huiral de $M$. pyrifera 
de bahía Porvenir, Tierra del Fuego, Hockey (1988) plantea que la gaviota común Larus dominicanus ("kelp gull") preda sobre Gaimardia sp., una de las especies más conspicuas sobre las frondas de la macroalga en el extremo sur de Sudamérica (Adami \& Gordillo 1999, Dayton 1985). Hockey (1988) determinó en su estudio una densidad de 5,3 gaviotas por $100 \mathrm{~m}^{2}$, con un consumo diario de Gaimardia sp. cercano a los 525.000 individuos. Esta cifra corresponde a una remoción de energía de ca. 21 $\mathrm{kJ} * \mathrm{~m}^{-2 *} \mathrm{~d}^{-1}$, cantidad estimada como muy por encima de la energía que remueven otros depredadores de alto nivel trófico asociados a M. pyrifera.

En un estudio reciente sobre la selección de hábitat y los patrones conductuales del delfín Lagenorhynchus australis en el estrecho de Magallanes, Viddi \& Lescrauwaet (2005) encontraron que la especie exhibe una significativa concentración en sólo una pequeña parte de área estudiada, la cual estuvo fuertemente asociada con huirales de $\mathrm{Ma}$ crocystis pyrifera. La conducta más frecuentemente observada en los huirales fue la de alimentación, principalmente dentro y en los bordes de ellos. La preferencia por este tipo de hábitat, que parece ser su principal territorio de alimentación, fue evidente durante todo el estudio. Los autores concluyen que $M$. pyrifera constituye el hábitat fundamental de $L$. australis en los sistemas costeros de la región, razón por la cual su protección es clave para la conservación de las poblaciones de la especie.

Palacios \& Mansilla (2003) describieron y evaluaron el desarrollo de gametófitos y esporófitos de $M$. pyrifera bajo condiciones de laboratorio a partir de muestras de poblaciones naturales de Tierra del Fuego. La población estudiada muestra abundantes zoosporas observables durante todo el año. De acuerdo a los resultados obtenidos, los autores sugieren que las poblaciones de la macroalga presentes en Magallanes podrían presentar un comportamiento reproductivo diferente a las poblaciones del resto del país. La variabilidad en aspectos reproductivos también fue notada en el estudio de Dayton (1985).

En relación con el potencial de utilización de Macrocystis pyrifera como recurso económico, Romo et al. (1984) estudiaron el efecto sobre el crecimiento de distintos tipos de poda sobre plantas individuales. El tipo de poda estudiada menos dañina sería la realizada $1 \mathrm{~m}$ por debajo del dosel, ya que podas más drásticas (1,5 m sobre el grampón de fijación o directamente sobre él) conducen a una alta mortalidad y bajo rendimiento de los especímenes.

\section{AGRADECIMIENTOS}

Este trabajo forma parte de una detallada línea base biológica establecida para un estudio de impacto ambiental realizado en isla Riesco, región de Magallanes. Fue realizado bajo las normas establecidas en el contrato suscrito entre Golder S.A. y la Universidad de Magallanes a través del Instituto de la Patagonia. Su publicación fue autorizada expresamente por Golder S.A.

\section{LITERATURA CITADA}

Adami, M. L. \& S. Gordillo 1999. Structure and dynamics of the biota associated with Macrocystis pyrifera (Phaeophyta) from the Beagle Channel, Tierra del Fuego. Sci. Mar. 63(Supl. 1): 183-191.

Alveal, K., H. Romo \& M. Avila 1973. Consideraciones ecológicas de las regiones de Valparaíso y de Magallanes. Rev. Biol. Mar, Valparaíso 15: 1-29.

Arnaud, P. M. 1974. Contribution a la bionomie marine benthique des regions antarctiques et subantartiques. Téthys 6: 467-653.

Arntz, W. E. 1999. Magellan - Antarctic: Ecosystems that drifted apart. Summary Review. Sci. Mar. 63 (Supl. 1): 503-511.

Castilla, J. C. 1985. Food webs and functional aspects of the kelp, Macrocystis pyrifera, community in the Beagle Channel, Chile. In: Siegfried W.R., P.R. Condy \& R.M. Laws (eds.) Antarctic Nutrient Cycles and Food Webs. pp 407-414. Springer-Verlag, Berlin, Heidelberg.

Castilla, J. C. \& C. A. Moreno 1982. Sea urchin and Macrocystis pyrifera: experimental test of their ecological relations in southern Chile. In: Lawrence J.M. (ed.) International Echinoderms Conference. pp 257-263. AA Balkema, Rotterndam.

Cariceo, Y., E. Mutschke \& C. Ríos 2002. Ensambles de Isopoda (Crustacea) en discos de fijación del alga Macrocystis pyrifera (L.) C. Agardh (Phaeophyta) en el estrecho de Magallanes, 
Chile. Anales Instituto Patagonia Serie Cs.

Nat. (Chile) 30:83-94

Dayton, P. K. 1974. Kelp communities of southern South America. Antarctic Journal of the U.S. IX(1): 22.

Dayton, P. K. 1985. The structure and regulation of some South American kelp communities. Ecol. Monogr. 55: 447-468.

Hockey, P. A. R. 1988. Kelp gulls Larus dominicanus as predators in kelp Macrocystis pyrifera beds. Oecologia 76: 155-157

Hoffmann, A. \& B. Santelices 1997. Marine flora of Central Chile. Ediciones Universidad Católica de Chile, Santiago. 434 pp.

Kühnemann, O. 1970 Algunas consideraciones sobre los bosques de Macrocystis pyrifera. Physis 29: 273-296.

Moreno, C. A. \& H. F. Jara 1984. Ecological studies on fish fauna associated with Macrocystis pyrifera belts in the south of Fueguian Islands, Chile. Mar. Ecol. Prog. Ser. 15: 99-107.

Morrison, E. \& G. Pequeño 2003. Primer registro del zoárcido Crossostomus sobrali (Lloris y Rucabado 1987) (Osteichthyes, Zoarcidae) en el estrecho de Magallanes, distrito fueguino, Chile. Anales Instituto Patagonia (Chile) 31: 105-109.

Ojeda, P. \& B. Santelices 1984. Invertebrate communities in hodfasts of the kelp Macrocystis pyrifera from southern Chile. Mar. Ecol. Prog. Ser. 16: 65-73.

Mansilla, A. 2006. Utilización de harina de la macroalga Macrocystis pyrifera como ingrediente para la alimentación de salmónidos en la región de Magallanes y Antártica Chilena. Publicación del Centro de Estudios Regionales UMAG, Primer Semestre 2006. Universidad de Magallanes. pp. 65-70.

Palacios, M. \& A. Mansilla 2003. Desarrollo de gametofitos y esporofitos de Macrocystis pyrifera (L.) C. Agardh (Laminariales: Lessoniaceae) de la Región de Magallanes en condiciones de laboratorio. Anales Instituto Patagonia (Chile) 31: 43-53.

Ríos, C., W. E. Arntz, D. Gerdes, E. Mutschke \& A. Montiel 2007. Spatial and temporal variability of the benthic assemblages associated to the holdfasts of the kelp Macrocystis pyrifera in the Straits of Magellan, Chile. Polar Biol. 31: 89-100.

Ríos, C., E. Mutschke \& Y. Cariceo 2003. Estructura poblacional de Pseudechinus magellanicus (Philippi 1857) (Echinoidea: Temnopleuridae) en grampones de la macroalga sublitoral $\mathrm{Ma}$ crocystis pyrifera (L.) Agardh en el estrecho de Magallanes. Anales Instituto Patagonia (Chile) 31: 75-86.

Romo, H., K. Alveal \& M. Avila 1984. El efecto de la poda en sobrevivencia, tamaño y rendimiento de Macrocystis pyrifera (L.) Ag. (Lessoniaceae) de isla Navarino, Chile. Gayana Botanica 41 (1-2): 127-135.

Santelices, B. \& F. P. Ojeda 1984a. Effects of canopy removal on the understory algal community structure of coastal forests of Macrocystis pyrifera from southern South America. Mar. Ecol. Prog. Ser. 14: 165-173.

Santelices, B. \& F. P. Ojeda 1984b. Population dynamics of coastal forests of Macrocystis pyrifera in Puerto Toro, Isla Navarino, Southern Chile. Mar. Ecol. Prog. Ser. 14: 175-183.

Santelices, B. 1992. Interacciones ecológicas en praderas de Macrocystis pyrifera de la región subantártica chilena. En: Gallardo V.A., O. Ferretty \& H. Moyano (eds.) Oceanografía en Antártica. pp. 501-507. ENEA-Proyecto Antártico-Italia, Centro EULA-U. de Concepción.

Searles, R. B., G. L. Leister \& J. F. Brauner 1974. Seaweds of southern South America. Antarctic Journal of the U.S. IX(1): 23-24

Vásquez, J. A., J. C. Castilla \& B. Santelices 1984. Distributional patterns and diets of four species of sea urchins in giant kelp forest (Macrocystis pyrifera) of Puerto Toro, Navarino Island, Chile. Mar. Ecol. Prog. Ser. 19: 55-63.

Vásquez, J. S. \& J. C. Castilla 1984. Some aspects of the biology and trophic range of Cosmasterias lurida (Asteroidea; Asteriidae) in belts of Macrocystis pyrifera at Puerto Toro. Medio Ambiente (Chile) 7: 47-51.

Viddi, F. A. \& A.K. Lescrauwaet 2005. Insights on habitat selection and behavioural patterns of Phale's dolphin (Lagenorhynchus australis) in the Strait of Magellan, southern Chile. Aquat. Mammals 31(2): 176-183. 